\title{
水溶性铑膦配合物插层水滑石的制备及主、客体组成对 其催化性能的影响
}

\author{
张娴, 石淑先, 陆军, 卫敏“ \\ 北京化工大学化工资源有效利用国家重点实验室, 北京 100029 \\ *联系人, E-mail: weimin@mail.buct.edu.cn
}

2007-09-03 收稿, 2007-12-03 接受

国家自然科学基金(批准号: 20601001)、新世纪优秀人才支持计划(批准号: NCET-05-121)、高等学校学科创新引智计划(批准号: B07004) 以及长江学者和创新团队发展计划资助项目

摘要 将水溶性铑膦配合物插层到层状材料水滑石层间, 用于烯烃氢甲酰化反应催 化剂. 采用 X 射线衍射(XRD), 傅里叶交换红外(FT-IR)和等离子耦合原子发射光谱 (ICP-AES)等手段对催化剂样品的晶相结构及组成进行了详细表征, 结果表明成功 制备了 $\mathrm{RhCl}-(\mathrm{CO})(\mathrm{TPPTS})_{2}\left(\mathrm{P}\left(m-\mathrm{C}_{6} \mathrm{H}_{4} \mathrm{SO}_{3} \mathrm{Na}\right)_{3}\right.$, 简称 TPPTS $)$ 和 TPPTS 共插层水滑石. 进一步研究了水滑石主体层板的组成及客体无机阴离子种类的改变对插层结构水 滑石催化剂样品在烯烃氢甲酰化反应中的催化性能的影响.

关键词

水滑石

烯烃氢甲酰化反应

负载催化剂

$\mathrm{RhCl}(\mathrm{CO})(\mathrm{TPPTS})_{2}$
烯烃氢甲酰化反应, 是指烯烃与合成气( $\mathrm{CO}$ 和 $\mathrm{H}_{2}$ ) 在催化剂的作用下生成多一个碳原子的正构醛和 异构醛的反应, 是当今最重要的合成气反应之一 ${ }^{[1]}$. 目前石油工业中普遍使用过渡金属作为这一反应的 催化剂, 它以每年 700 万吨醛的产量成为当今最重 要的有机化工生产工艺之一 ${ }^{[2]}$. 近几十年来, 烯烃氢 甲酰化催化剂的固载化已经越来越引起人们的关注 [3,4]，它具有与均相催化剂相近的活性，相近或稍好 的选择性, 且催化剂与产物容易分离, 不流失 ${ }^{[5]}$ 等优 点. 据大量文献及专利报道, 将铑等贵金属负载到 $\mathrm{SiO}_{2}$ 及其他氧化物载体上 ${ }^{[6,7]}$, 得到具有较好催化性 能的烯烃氢甲酰化催化剂.

水滑石(layered double hydroxides, LDHs)是由层 间阴离子及带正电荷层板堆积而成的化合物, 其结 构类似于水镁石 $\mathrm{Mg}(\mathrm{OH})_{2}$, 组成通式为: $\left[\mathrm{M}^{\prime \prime}{ }_{1-x} \mathrm{M}^{\mathrm{III}}{ }_{x^{-}}\right.$ $\left.(\mathrm{OH})_{2}\right]^{x+}\left(\mathrm{A}^{n-}\right)_{x / n} \cdot y \mathrm{H}_{2} \mathrm{O}$, 其中 $\mathrm{M}^{2+}$ 和 $\mathrm{M}^{3+}$ 分别为二价 和三价金属阳离子; $\mathrm{A}^{n-}$ 是层间阴离子, $x$ 为 $\mathrm{M}^{3+} /\left(\mathrm{M}^{2+}+\right.$ $\mathrm{M}^{3+}$ )摩尔比 ${ }^{[8 \sim 10]}$. LDHs层状材料的一个重要特性就 是其层间阴离子的可交换性及主体层板和层间客体 的可调控性, 从而可以形成一系列新的功能材料, 在
离子交换和吸附 ${ }^{[11]}$ 、分子容器及分子反应器 ${ }^{[12]}$ 、医 药 [13,14]等方面的应用研究已取得了很大进展, 尤其在 催化领域的应用更为广泛. Choudary等人 ${ }^{[15]}$ 利用离子 交换法将 $\mathrm{OsO}_{4}{ }^{2-}$ 负载到LDHs层间用于烯烃不对称二 羟基化反应，体现了较高的活性; Bhattacharjee等人 ${ }^{[16]}$ 将手性锰( III)的三磺酸盐配合物插入 ZnAl- LDHs 层间, 成功得到了一种稳定的、活性及选择性较好的 非均相环氧化催化剂; 其他还有 $\mathrm{LDH}-\mathrm{WO}_{4}$ 催化氧化 溴化反应 ${ }^{[17]}$ 的报道.

在我们的前期工作中 ${ }^{[18]}$, 以水溶性铑膦配合物 $\mathrm{RhCl}(\mathrm{CO})(\mathrm{TPPTS})_{2}\left(\mathrm{P}\left(m-\mathrm{C}_{6} \mathrm{H}_{4} \mathrm{SO}_{3} \mathrm{Na}\right)_{3}\right.$, 简称 TPPTS $)$ 和TPPTS为插层客体, 水滑石类阴离子层状化合物为 主体, 采用原位络合法制备水溶性铑膦配合物插层 LDHs，并将其作为烯烃氢甲酰化反应的负载催化剂; 对比研究了该法制备的催化剂与相应的水油两相催 化剂的性能差异, 给出了合理的解释. 本文在前期工 作的基础上, 继续采用原位络合法制备 $\mathrm{RhCl}(\mathrm{CO})$ $(\mathrm{TPPTS})_{2}$ 插层LDHs, 着重研究该催化剂中主、客体 因素(包括主体层板 $\mathrm{M}^{2+}$ 元素及层间共存无机阴离子 种类)对其在烯烃氢甲酰化反应中催化行为的影响, 
进一步揭示主客体相互作用与催化性能之间的关系.

\section{1 实验}

(i ) 药品及试剂. TPPTS(四川大学提供), $\mathrm{RhCl}_{3}$. $3 \mathrm{H}_{2} \mathrm{O}$ (有色金属研究总院, 含 $\mathrm{Rh} 40 \%$ ), 其余试剂均 为分析纯, $\mathrm{H}_{2} \mathrm{O}$ 为去离子水, 经去二氧化碳处理.

(ii ) LDHs前体的制备. 参照文献以单滴法制 备无机阴离子插层LDHs离子交换前体: 硝酸根锌铝 $\mathrm{LDHs}^{[19]}$, 硝酸根镍铝 $\mathrm{LDHs}^{[20]}$, 氯离子锌铝 $\mathrm{LDHs}^{[21]}$, 硫酸根锌铝 LDHs ${ }^{[22]}$. 离子交换法制备 TPPTS插层 LDHs原位络合前体: 取一定量TPPTS溶于 $100 \mathrm{~mL}$ 脱 $\mathrm{CO}_{2}$ 的去离子水中, 加入一定量无机阴离子插层 LDHs离子交换前体, $\mathrm{N}_{2}$ 保护下于 $80^{\circ} \mathrm{C} 反$ 应 $72 \mathrm{~h}$, 然 后抽滤、水洗, 室温真空干燥 $48 \mathrm{~h}$, 得到TPPTS插层 LDHs原位络合前体.

(iii) 原位络合法制备水溶性铑膦配合物插层 LDHs. 采用原位络合法 ${ }^{[18]}$ 制备水溶性铑膦配合物 插层LDHs. 将一定量的 $\mathrm{RhCl}_{3} \cdot 3 \mathrm{H}_{2} \mathrm{O}$ 溶于 $10 \mathrm{~mL}$ 无水 乙醇中, 通入 $\mathrm{CO}$ 气体, 并升温至 $78^{\circ} \mathrm{C}$, 回流反应 50 $\mathrm{min}$, 停止加热. 滴加TPPTS/A-M $\mathrm{M}_{2} \mathrm{Al}-\mathrm{LDHs}$ 分散于少 量水的悬浊液, 继续在 $78^{\circ} \mathrm{C}$ 回流反应 $90 \mathrm{~min}$. 冷却并 加入 $40 \mathrm{~mL}$ 无水乙醇, 继续冷却至 $20^{\circ} \mathrm{C}$ 过滤, 用乙醇 洗涤得到的黄色沉淀至中性. 真空 $60^{\circ} \mathrm{C}$ 干燥, 得淡黄 色固体. 产物编号 $\mathrm{RhP}_{2} / \mathrm{P} / \mathrm{A}-\mathrm{M}_{2} \mathrm{Al}-\mathrm{LDHs}\left(\mathrm{RhP}_{2}\right.$ 指 RhCl$(\mathrm{CO})(\mathrm{TPPTS})_{2}, \mathrm{P}$ 指TPPTS; $\mathrm{A}$ 为无机阴离子 $\mathrm{NO}_{3}^{-}, \mathrm{Cl}^{-}$, $\mathrm{SO}_{4}^{2-}, \mathrm{M}$ 为层板金属 $\mathrm{Zn}, \mathrm{Ni}$ ).

(iv) 烯烃氢甲酰化反应. 将一定量的铑膦配合 物插层 $\mathrm{LDHs}$ 催化剂样品 $\mathrm{RhP}_{2} / \mathrm{P} / \mathrm{A}-\mathrm{M}_{2} \mathrm{Al}-\mathrm{LDHs}$, 反 应底物 1 -己烯 $1 \mathrm{~mL}$, 溶剂甲苯 $2 \mathrm{~mL}$, 三乙胺 $0.05 \mathrm{~mL}$, 置于高压反应金中, 通入等摩尔比 $\mathrm{CO} / \mathrm{H}_{2}$ 的混合气 体置换 5 次, 然后充入等摩尔比 $\mathrm{CO} / \mathrm{H}_{2}$ 混合气体至预 定压力 $1.6 \mathrm{MPa}$ 后, 加热升温到反应温度 $100^{\circ} \mathrm{C}$, 开 始磁力搅拌. 反应 $6 \mathrm{~h}$ 后, 用冰水浴迅速冷却取出, 离心分离催化剂样品及烯烃氢甲酰化反应产物. 采 用 GC-MS 对液相产物组成进行定量分析.

（v）表征方法及仪器. 日本岛津 XRD-6000 型 $\mathrm{X}$ 射线衍射仪 $(\mathrm{CuK \alpha}$ 射线), 用于表征样品的晶体结 构. 德国 Bruker VECTOR 22 型红外光谱仪, 用于对 样品组成结构进行定性分析. 日本岛津 ICPS-7500 型 元素分析仪对样品的 $\mathrm{Zn}, \mathrm{Al}, \mathrm{Rh}, \mathrm{P}, \mathrm{S}$ 元素含量进行定 量分析. 日本岛津 2010 GC-MS 联用仪, 采用程序升 温的方法, 用于烯烃氢甲酰化反应产物进行分离与
检测.

\section{2 结果与讨论}

2.1 LDHs 主体层板 $\mathrm{M}^{2+}$ 元素对插层 LDHs 样品催化 性能的影响

基于 LDHs 所特有的主体层板可调控性, 通过改 变 $\mathrm{M}^{2+}$ 金属元素以制备不同 LDHs 载体性质的催化剂 样品 $\mathrm{RhP}_{2} / \mathrm{P}-\mathrm{Zn}_{2} \mathrm{Al}-\mathrm{LDHs}$ 和 $\mathrm{RhP}_{2} / \mathrm{P}-\mathrm{Ni}_{2} \mathrm{Al}-\mathrm{LDHs}$. 利 用 XRD, FT-IR 及 ICP-AES 等表征手段对样品的晶相 结构及组成进行考察, 并对催化剂样品在烯烃氢甲 酰化反应中的催化性能进行对比研究.

图 1 给出了样品 $\mathrm{RhP}_{2} / \mathrm{P}-\mathrm{Zn}_{2} \mathrm{Al}-\mathrm{LDHs}$ 和 $\mathrm{RhP}_{2} / \mathrm{P}-$ $\mathrm{Ni}_{2} \mathrm{Al}-\mathrm{LDHs}$ 的粉末 X射线衍射谱图，表 1 列出了相应 的层间距及晶胞参数. 由XRD谱图可以看到样品 $\mathrm{RhP}_{2} / \mathrm{P}-\mathrm{Zn}_{2} \mathrm{Al}-\mathrm{LDH}$ 和 $\mathrm{RhP}_{2} / \mathrm{P}-\mathrm{Ni}_{2} \mathrm{Al}-\mathrm{LDHs}$ 均具有典 型的LDHs特征衍射峰 ${ }^{[23,24]}$, 但是同文献中报道的有 机阴离子插层产物一样 ${ }^{[25]}$, 其中的一些 $(h k l)$ 衍射峰 消失或宽化. $\mathrm{RhP}_{2} / \mathrm{P}-\mathrm{Zn}_{2} \mathrm{Al}-\mathrm{LDH}$ 和 $\mathrm{RhP}_{2} / \mathrm{P}-\mathrm{Ni}_{2} \mathrm{Al}$ LDHs样品的层间距 $d_{003}$ 值分别为 1.579 和 $1.595 \mathrm{~nm}$ (表 1), 与各自的离子交换前体(硝酸根LDHs)相比较 分别增加了 0.701 和 $0.697 \mathrm{~nm}$, 层间距的显著增加说

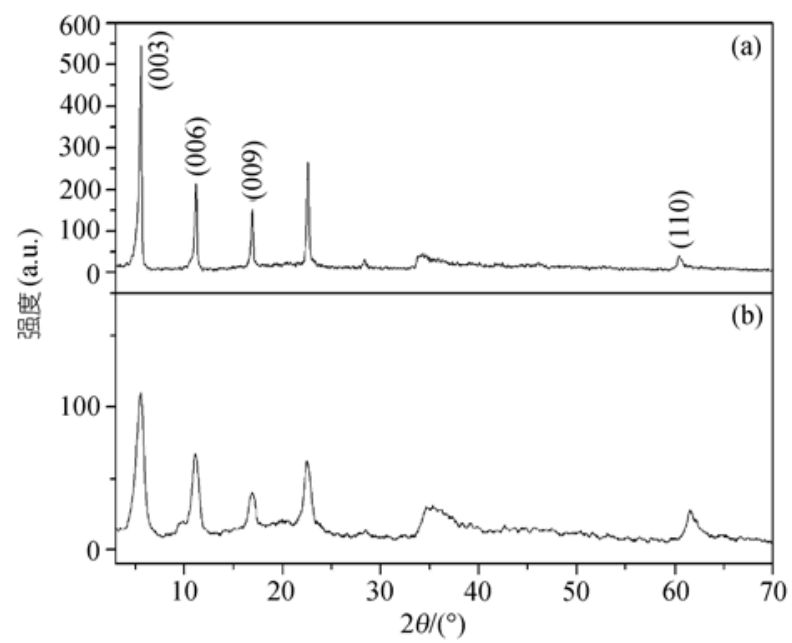

图 1 水溶性铑膦配合物插层 LDHs 样品的 $\mathrm{X}$ 射线衍射图 (a) $\mathrm{RhP}_{2} / \mathrm{P}-\mathrm{Zn}_{2} \mathrm{Al}-\mathrm{LDHs}$; (b) $\mathrm{RhP}_{2} / \mathrm{P}-\mathrm{Ni}_{2} \mathrm{Al}-\mathrm{LDHs}$

表 1 水溶性铑膦配合物插层 LDHs 样品的晶胞参数

\begin{tabular}{ccc}
\hline 样品 & $\mathrm{RhP}_{2} / \mathrm{P}-\mathrm{Zn}_{2} \mathrm{Al}-\mathrm{LDHs}$ & $\mathrm{RhP}_{2} / \mathrm{P}-\mathrm{Ni}_{2} \mathrm{Al}-\mathrm{LDHs}$ \\
\hline$d_{003} / \mathrm{nm}$ & 1.579 & 1.595 \\
$d_{110} / \mathrm{nm}$ & 0.153 & 0.151 \\
晶胞参数 $a / \mathrm{nm}$ & 0.306 & 0.302 \\
晶胞参数 $c / \mathrm{nm}$ & 4.737 & 4.785 \\
\hline
\end{tabular}


明成功制备了有机阴离子插层 LDHs 样品, 并且二者 具有相似的插层组装结构. 但是与 $\mathrm{RhP}_{2} / \mathrm{P}-\mathrm{Zn}_{2} \mathrm{Al}$ LDHs 相比, $\mathrm{RhP}_{2} / \mathrm{P}-\mathrm{Ni}_{2} \mathrm{Al}-\mathrm{LDHs}$ 的衍射峰强度明显降 低, 半峰宽增大, 表明前者的晶形规整度高于后者.

图 2 为 TPPTS, $\mathrm{RhCl}(\mathrm{CO})(\mathrm{TPPTS})_{2}$ 及其插层 $\mathrm{LDHs}$ 样品的FT-IR谱图. TPPTS和 RhCl(CO)(TPPTS $)_{2}$ 的 FT-IR谱图(图 2(a)和(b))与文献报道一致 [26]. 铑膦配 合物 $\mathrm{RhCl}(\mathrm{CO})(\mathrm{TPPTS})_{2}$ 中磺酸根的特征吸收峰 $v_{\mathrm{oso}}$, $v_{\mathrm{so}}$ 和 $v_{\mathrm{so}}^{\prime}$ 出现在 $1196,1040,628 \mathrm{~cm}^{-1}$ (图 2(b)), 而插层 样品 $\mathrm{RhP}_{2} / \mathrm{P}-\mathrm{Zn}_{2} \mathrm{Al}$-LDHs向低波移动至 $1182 ， 1036$, $619 \mathrm{~cm}^{-1}$ (图 2(c)). 磺酸根特征吸收峰向低波数移动 是由于层间阴离子的磺酸根与LDHs层板间的静电引 力作用造成的. 另一方面, 也可能是磺酸根中氧原子 与层板 $\mathrm{Al}^{3+}$ 的配位作用造成 $\mathrm{S}-\mathrm{O}$ 键的电荷密度降低 从而导致其力常数减小, 造成了 $\mathrm{S}-\mathrm{O}$ 键的振动吸收 峰向低波数移动, 这与文献中报道的 2,6-二磺酸菜盐 ${ }^{[27]}$ 和茜素磺酸钠盐插层LDHs ${ }^{[28]}$ 后的FT-IR变化一致. 与 $\mathrm{RhP}_{2} / \mathrm{P}-\mathrm{Zn}_{2} \mathrm{Al}-\mathrm{LDHs}$ 相比, 样品 $\mathrm{RhP}_{2} / \mathrm{P}-\mathrm{Ni}_{2} \mathrm{Al}$ LDHs中磺酸根的 3 个特征吸收峰发生了更大程度的 移动: 向低波数移动至 $1178,1035,618 \mathrm{~cm}^{-1}$ (图 2(d)). 这说明不同主体层板与插层客体阴离子的相互作用 存在差异 ${ }^{[29,30]}$ ，这与层板金属的电负性相关. $\mathrm{Zn}$ 及 Ni 元素的电负性分别为 1.6 及 $1.9^{[31]}$, 电负性的增强使 得主体层板中 $\mathrm{M}^{2+}$ 的给电子能力降低, 这一结果一方 面会造成层间客体阴离子中磺酸根的 $\mathrm{O}$ 与层板差基 - OH所形成的氢键作用增强; 另一方面会使相邻 $\mathrm{M}^{3+}$ 周围的正电荷密度增加, 进而引起 $\mathrm{M}^{3+}$ 与客体阴 离子的静电引力增强. 因此, $\mathrm{RhP}_{2} / \mathrm{P}-\mathrm{Ni}_{2} \mathrm{Al}-\mathrm{LDHs}$ 样

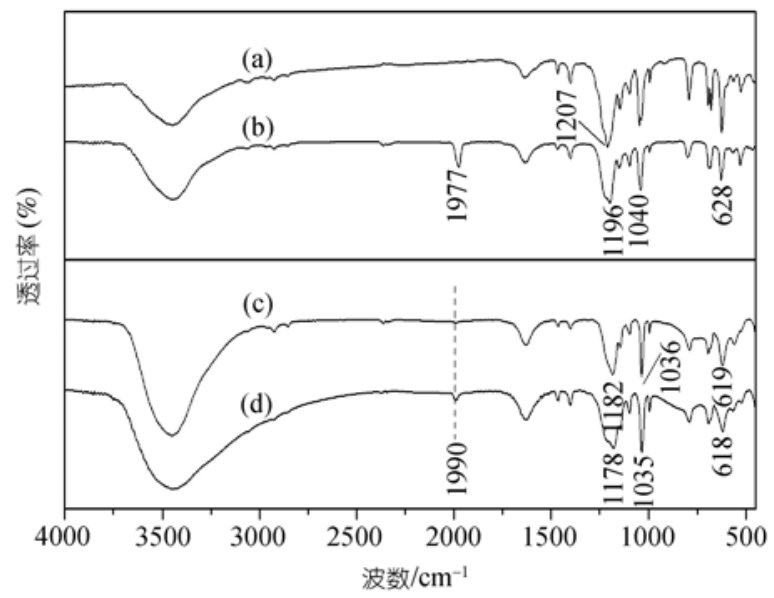

图 2 水溶性铑膦配合物插层 LDHs 样品的红外光谱

(a) TPPTS; (b) RhCl(CO)(TPPTS) $)_{2}$; (c) $\mathrm{RhP}_{2} / \mathrm{P}_{-} \mathrm{Zn}_{2} \mathrm{Al}-\mathrm{LDHs}$; (d) $\mathrm{RhP}_{2} / \mathrm{P}-\mathrm{Ni}_{2} \mathrm{Al}-\mathrm{LDHs}$
品中的主-客体相互作用力比 $\mathrm{RhP}_{2} / \mathrm{P}-\mathrm{Zn}_{2} \mathrm{Al}-\mathrm{LDHs}$ 强. 这种不同主体层板 $\mathrm{M}^{2+}$ 元素所导致的主-客体相互作 用的变化也使得产物的层间距略有不同, 如表 1 所示. 样品 $\mathrm{RhP}_{2} / \mathrm{P}-\mathrm{Zn}_{2} \mathrm{Al}-\mathrm{LDHs}$ 和 $\mathrm{RhP}_{2} / \mathrm{P}-\mathrm{Ni}_{2} \mathrm{Al}-\mathrm{LDHs}$ 的 $v_{\mathrm{CO}}$ 振动吸收峰与 $\mathrm{RhCl}(\mathrm{CO})(\mathrm{TPPTS})_{2}\left(1977 \mathrm{~cm}^{-1}\right)$ 相 比较向高波数移动(图 2(b) (d)), 均出现在 $1990 \mathrm{~cm}^{-1}$, 这可能是层间磺酸根与 LDHs 层板间的相互作用力 间接导致的. 同时, 样品 $\mathrm{RhP}_{2} / \mathrm{P}-\mathrm{Zn}_{2} \mathrm{Al}-\mathrm{LDHs}$ 和 $\mathrm{RhP}_{2} /$ $\mathrm{P}-\mathrm{Ni}_{2} \mathrm{Al}-\mathrm{LDHs}$ 分别在 $1464,1398 \mathrm{~cm}^{-1}$ 附近出现了与 $\mathrm{P}$ 连接的苯环 $\mathrm{C}=\mathrm{C}$ 环伸缩振动的特征吸收峰, 788, $692 \mathrm{~cm}^{-1}$ 附近出现了苯环的 $\mathrm{C}-\mathrm{H}$ 面外弯曲振动的特 征吸收峰 (图 2(c),(d)), 与 $\mathrm{RhCl}(\mathrm{CO})(\mathrm{TPPTS})_{2}$ 和 TPPTS 的相应特征吸收峰 $\left(1466,1398 \mathrm{~cm}^{-1} ; 791,692\right.$ $\mathrm{cm}^{-1}$ )相比未发生位移(图 2(a),(b)).

对样品的元素含量进行了 ICP-AES 测试, 并计 算了主要元素的摩尔比, 结果列于表 2. ICP-AES 结 果(表2)进一步确认了插层结构 LDHs 样品的组成, 结 合 XRD 及 FT-IR 结果可以得出, $\mathrm{RhCl}(\mathrm{CO})(\mathrm{TPPTS})_{2}$ 与 TPPTS 已共插层进入锌铝、镍铝 LDHs 层间, 成功 制备了插层 LDHs 催化剂.

\section{表 2 水溶性铑膦配合物插层 LDHs 样品中主要元素的} 含量及摩尔比

\begin{tabular}{ccc}
\hline 样品 & $\mathrm{RhP}_{2} / \mathrm{P}-\mathrm{Zn}_{2} \mathrm{Al}-\mathrm{LDHs}$ & $\mathrm{RhP}_{2} / \mathrm{P}-\mathrm{Ni}_{2} \mathrm{Al}-\mathrm{LDHs}$ \\
\hline 摩尔比 $\mathrm{M} / \mathrm{Al}$ & 2.0 & 1.6 \\
摩尔比 $\mathrm{P} / \mathrm{Rh}$ & 18.4 & 16.2 \\
$\mathrm{Rh}(w / w)(\%)$ & 0.36 & 0.31 \\
\hline
\end{tabular}

在相同的烯烃氢甲酰化反应条件下考察了 LDHs 主体层板 $\mathrm{M}^{2+}$ 元素对样品 $\mathrm{RhCl}(\mathrm{CO})(\mathrm{TPPTS})_{2}$ 和 TPPTS 共插层 $\mathrm{LDHs}$ 催化性能的影响, 结果如表 3 .

由表 3 可以看到, 在近似的 $\mathrm{M}^{2+} / \mathrm{Al}$ 及 $\mathrm{P} / \mathrm{Rh}$ 摩尔比 条件下, 样品 $\mathrm{RhP}_{2} / \mathrm{P}-\mathrm{Zn}_{2} \mathrm{Al}-\mathrm{LDHs}$ 和 $\mathrm{RhP}_{2} / \mathrm{P}-\mathrm{Ni}_{2} \mathrm{Al}$ $\mathrm{LDHs}$ 在反应活性方面存在较大差别: 总转化率依次 为 $76 \%, 32 \%$, 产物醛的 TOF值分别为 $267,54 \mathrm{~h}^{-1}$ (表 3). 据文献 $[32,33]$ 报道，在该催化体系中，无论铑以 何种形式的前体加入, 在氢甲酰化反应条件下都会 很快形成 $\mathrm{HRh}(\mathrm{CO})_{m} \mathrm{~L}_{n}[\mathrm{~L}=\mathrm{TPPTS}, m, n=1,2,3]$ 型配合物 (图 3). 其中的催化活性物种为 $16 \mathrm{e}$ 络合物, 如 HRh-

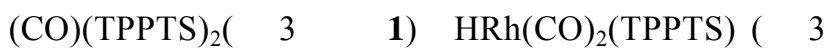
物质 3). 本文催化剂样品中的载体 LDHs层板由 $\mathrm{MO}_{6}$ 八面体组成, 具有一定的碱性 ${ }^{[34,35]}$. 而正是载体的这 种性质有利于催化活性物种的生成(图 3, 步骤 1), 即: 通过消耗产生的 $\mathrm{HC}$ 而促进催化活性物种的形成. 
表 3 LDHs 主体层板 $\mathrm{M}^{2+}$ 金属种类对催化剂样品在 1-己烯氢甲酰化反应中催化性能的影响 ${ }^{\text {a) }}$

\begin{tabular}{|c|c|c|c|c|}
\hline 样品 & 转化率(\%) & $\mathrm{TOF} / \mathrm{mol}\left(\right.$ 醛) $\cdot \mathrm{mol}^{-1}(\mathrm{Rh}) \cdot \mathrm{h}^{-1}$ & 醛选择性(\%) & $n / i$ \\
\hline $\mathrm{RhP}_{2} / \mathrm{P}-\mathrm{Zn}_{2} \mathrm{Al}-\mathrm{LDHs}$ & 75.94 & 266.97 & 84.38 & 2.19 \\
\hline $\mathrm{RhP}_{2} / \mathrm{P}-\mathrm{Ni}_{2} \mathrm{Al}-\mathrm{LDHs}$ & 32.38 & 54.31 & 40.27 & 1.58 \\
\hline
\end{tabular}

a) $\mathrm{Rh} / 1$-hexene $=4.0 \times 10^{-4}$, 反应条件: $1 \mathrm{~mL}$ 1-hexene, $1.6 \mathrm{MPa}, 100^{\circ} \mathrm{C}, n\left(\mathrm{H}_{2}\right) / n(\mathrm{CO})=1,6 \mathrm{~h}$ 反应时间

$$
\begin{aligned}
& \mathrm{RhCl}(\mathrm{CO})(\mathrm{TPPTS})_{2}+\mathrm{H}_{2} \underset{\text { 步骤1 }}{\rightleftharpoons} \mathrm{HRh}(\mathrm{CO})(\underset{1}{\mathrm{TPPTS}})_{2}+\mathrm{HCl} \\
& \text { 步骤 } 2 \underset{ \pm \mathrm{CO}}{1} \\
& \begin{array}{cc}
\mathrm{HRh}(\mathrm{CO})_{2}(\mathrm{TPPTS})_{2} \\
\mathbf{2}
\end{array} \stackrel{\text { 步骤3 }}{\underset{\text { TPPTS }}{\rightleftharpoons}} \mathrm{HRh}(\mathrm{CO})_{2} \text { (TPPTS) }
\end{aligned}
$$

\section{图 3 催化活性物种的生成过程}

不同的LDHs载体的碱性强弱与 $\mathrm{M}^{2+}$ 氢氧化物的碱性 强弱基本一致, 即与 $\mathrm{M}^{2+}$ 的电负性直接关联, 由此可 知 $\mathrm{RhP}_{2} / \mathrm{P}-\mathrm{Zn}_{2} \mathrm{Al}-\mathrm{LDHs}$ 的载体碱性比 $\mathrm{RhP}_{2} / \mathrm{P}-\mathrm{Ni}_{2} \mathrm{Al}-$ LDHs的强. 结合催化活性物种的生成过程及LDHs载 体碱性强弱的决定因素, 就能很好地解释两个催化 剂样品 $\mathrm{RhP}_{2} / \mathrm{P}-\mathrm{Zn}_{2} \mathrm{Al}-\mathrm{LDHs}$ 和 $\mathrm{RhP}_{2} / \mathrm{P}-\mathrm{Ni}_{2} \mathrm{Al}-\mathrm{LDHs}$ 在 反应活性方面的较大差异. 由于前者的LDHs载体碱 性较强, 有利于催化活性物种的生成(图 3 步骤 1), 因 此其催化活性较高. 我们的研究结果与文献报道的有 关催化剂活性与载体碱性的关系是一致的 $117,36,37]$.

样品 $\mathrm{RhP}_{2} / \mathrm{P}-\mathrm{Zn}_{2} \mathrm{Al}-\mathrm{LDHs}$ 和 $\mathrm{RhP}_{2} / \mathrm{P}-\mathrm{Ni}_{2} \mathrm{Al}-\mathrm{LDHs}$ 在反应选择性方面也有较大差别, 成醛选择性依次 为 $84 \%, 40 \%$, 醛的正/异构比分别为 2.2 和 1.6 , 这是 由两样品不同的主-客体作用力差异造成的. 由 FT-IR 可知, 样品 $\mathrm{RhP}_{2} / \mathrm{P}-\mathrm{Ni}_{2} \mathrm{Al}-\mathrm{LDHs}$ 中的主-客体相互作 用力比 $\mathrm{RhP}_{2} / \mathrm{P}-\mathrm{Zn}_{2} \mathrm{Al}-\mathrm{LDHs}$ 的强, 而较强的主-客体 相互作用造成层间 TPPTS 配体与催化活性物种 HRh$(\mathrm{CO})_{2}$ (TPPTS)(图 3 物质 3)接触的空间阻力较大, 客客体相互作用减弱, 不利于 $\mathrm{HRh}(\mathrm{CO})(\mathrm{TPPTS})_{2}$ (图 3 物质 1)的生成. 而 $\mathrm{HRh}(\mathrm{CO})(\mathrm{TPPTS})_{2}$ 的生成对于提 高醛选择性是至关重要的. 因此在主-客体作用力较 强的样品 $\mathrm{RhP}_{2} / \mathrm{P}-\mathrm{Ni}_{2} \mathrm{Al}-\mathrm{LDHs}$ 的催化体系中, 醛的化 学选择性和空间选择性均较低.

2.2 LDHs 层间无机离子种类对催化剂样品催化性 能的影响

在烯烃氢甲酰化反应两相催化体系中, 研究者 通常采用外加无机盐作为助催化剂提高催化剂活性 或选择性 ${ }^{[38]}$. 根据文献报道，同时结合LDHs制备的 特点, 通过改变制备TPPTS插层LDHs的离子交换前 体的组成, 制备含不同层间无机阴离子的催化剂样
品 $\mathrm{RhP}_{2} / \mathrm{P} / \mathrm{NO}_{3}-\mathrm{Zn}_{2} \mathrm{Al}-\mathrm{LDHs}, \mathrm{RhP}_{2} / \mathrm{P} / \mathrm{Cl}-\mathrm{Zn}_{2} \mathrm{Al}$-LDHs 和 $\mathrm{RhP}_{2} / \mathrm{P} / \mathrm{SO}_{4}-\mathrm{Zn}_{2} \mathrm{Al}-\mathrm{LDH}$, 利用 XRD, FT-IR 及 ICPAES 等表征手段对其晶相结构及组成进行研究. 同 时考察 LDHs 催化剂样品层间的无机阴离子种类对 其催化性能的影响.

图 4 给出了样品 $\mathrm{RhP}_{2} / \mathrm{P} / \mathrm{NO}_{3}-\mathrm{Zn}_{2} \mathrm{Al}-\mathrm{LDHs}, \mathrm{RhP}_{2} /$ $\mathrm{P} / \mathrm{Cl}-\mathrm{Zn}_{2} \mathrm{Al}-\mathrm{LDHs}$ 和 $\mathrm{RhP}_{2} / \mathrm{P} / \mathrm{SO}_{4}-\mathrm{Zn}_{2} \mathrm{Al}-\mathrm{LDHs}$ 的粉末 $\mathrm{X}$ 射线衍射谱图. 表 4 列出了相应的层间距及晶胞参数. $\mathrm{XRD}$ 结果(图 4)表明, 由不同无机阴离子插层前体得 到的 $\mathrm{RhP}_{2} / \mathrm{P} / \mathrm{NO}_{3}-\mathrm{Zn}_{2} \mathrm{Al}-\mathrm{LDHs}, \mathrm{RhP}_{2} / \mathrm{P} / \mathrm{Cl}-\mathrm{Zn}_{2} \mathrm{Al}-$ LDHs和 $\mathrm{RhP}_{2} / \mathrm{P} / \mathrm{SO}_{4}-\mathrm{Zn}_{2} \mathrm{Al}-\mathrm{LDHs}$ 均具有典型的LDHs 晶相结构特征 ${ }^{[23,24]}, d_{003}$ 值分别为 $1.571,1.555$ 和 $1.566 \mathrm{~nm}$, 与相应硝酸根、氯离子、硫酸根LDHs离子 交换前体的 $d_{003}$ 值相比分别增加了 $0.693,0.785$ 和 $0.496 \mathrm{~nm}$ ，层间距的显著增加说明成功制备了有机阴 离子插层 $\mathrm{LDHs}$ 样品. 样品 $\mathrm{RhP}_{2} / \mathrm{P} / \mathrm{Cl}-\mathrm{Zn}_{2} \mathrm{Al}-\mathrm{LDHs}$

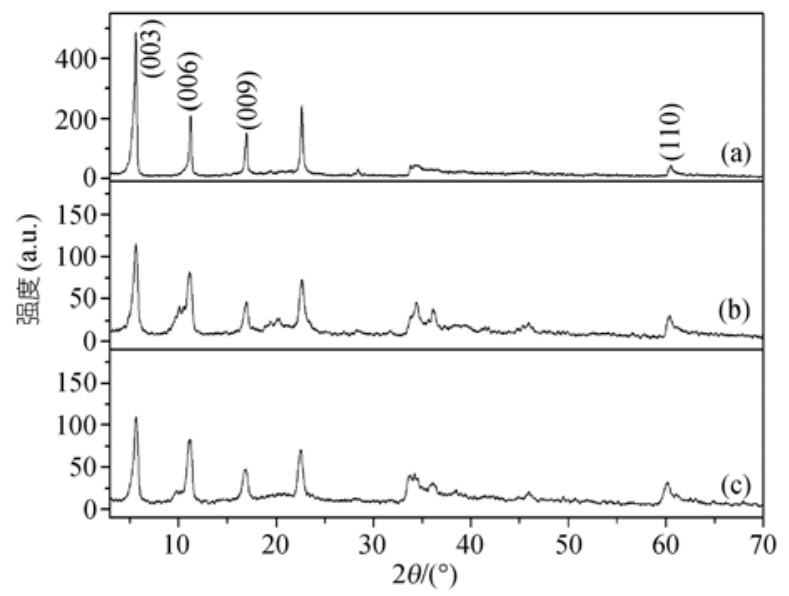

图 4 水溶性铑膦配合物插层 LDHs 样品的 $\mathrm{X}$ 射线衍射图 (a) $\mathrm{RhP}_{2} / \mathrm{P} / \mathrm{NO}_{3}-\mathrm{Zn}_{2} \mathrm{Al}-\mathrm{LDHs}$; (b) $\mathrm{RhP}_{2} / \mathrm{P} / \mathrm{Cl}-\mathrm{Zn}_{2} \mathrm{Al}-\mathrm{LDHs}$; (c) $\mathrm{RhP}_{2} / \mathrm{P} / \mathrm{SO}_{4}-\mathrm{Zn}_{2} \mathrm{Al}-\mathrm{LDHs}$ 
表 4 水溶性铑膦配合物插层 LDHs 样品的晶胞参数

\begin{tabular}{cccc}
\hline 样品 & $\mathrm{RhP}_{2} / \mathrm{P} / \mathrm{NO}_{3}-\mathrm{Zn}_{2} \mathrm{Al}-\mathrm{LDHs}$ & $\mathrm{RhP}_{2} / \mathrm{P} / \mathrm{Cl}-\mathrm{Zn}_{2} \mathrm{Al}-\mathrm{LDHs}$ & $\mathrm{RhP}_{2} / \mathrm{P} / \mathrm{SO}_{4}-\mathrm{Zn}_{2} \mathrm{Al}_{-}-\mathrm{LDHs}$ \\
\hline$d_{003} / \mathrm{nm}$ & 1.571 & 1.555 & 1.566 \\
$d_{110} / \mathrm{nm}$ & 0.153 & 0.153 & 0.153 \\
晶胞参数 $a / \mathrm{nm}$ & 0.306 & 0.306 & 0.306 \\
晶胞参数 $c / \mathrm{nm}$ & 4.713 & 4.665 & 4.698 \\
\hline
\end{tabular}

和 $\mathrm{RhP}_{2} / \mathrm{P} / \mathrm{SO}_{4}-\mathrm{Zn}_{2} \mathrm{Al}-\mathrm{LDHs}$ 的衍射峰强度明显低于 $\mathrm{RhP}_{2} / \mathrm{P} / \mathrm{NO}_{3}-\mathrm{Zn}_{2} \mathrm{Al}-\mathrm{LDHs}$ ，并且前两者出现宽化的现 象, 这说明 $\mathrm{RhP}_{2} / \mathrm{P} / \mathrm{NO}_{3}-\mathrm{Zn}_{2} \mathrm{Al}-\mathrm{LDHs}$ 样品的晶型最为 规整.

对样品 $\mathrm{RhP}_{2} / \mathrm{P} / \mathrm{NO}_{3}-\mathrm{Zn}_{2} \mathrm{Al}-\mathrm{LDHs}, \mathrm{RhP}_{2} / \mathrm{P} / \mathrm{Cl}$ $\mathrm{Zn}_{2} \mathrm{Al}-\mathrm{LDHs}$ 和 $\mathrm{RhP}_{2} / \mathrm{P} / \mathrm{SO}_{4}-\mathrm{Zn}_{2} \mathrm{Al}-\mathrm{LDHs}$ 进行 FT-IR 表征, 结果如图 5 所示. 3 个样品的磺酸根特征吸收 峰 $v_{\mathrm{oso}}, v_{\mathrm{so}}$ 和 $v_{\mathrm{so}}^{\prime}$ 与 TPPTS 和 $\mathrm{RhCl}(\mathrm{CO})(\mathrm{TPPTS})_{2}$ (图 2(a) 和(b))相比向低波数移动至 1182,1036 和 $619 \mathrm{~cm}^{-1}$ 附 近(图 5), 由此可以推断层间客体阴离子主要通过磺 酸根与主体层板发生作用而存在于层间. 三者的 $v_{\mathrm{CO}}$ 振动吸收峰(图 5)相比 $\mathrm{RhCl}(\mathrm{CO})(\mathrm{TPPTS})_{2}$ (图 2(b))向 高波数发生了移动, 均出现在 $1990 \mathrm{~cm}^{-1}$. 同时, 样品 与 $\mathrm{P}$ 连接的苯环 $\mathrm{C}=\mathrm{C}$ 环伸缩振动的特征吸收峰及 苯环的 $\mathrm{C}-\mathrm{H}$ 面外弯曲振动等特征吸收峰, 与 $\mathrm{RhCl}-$

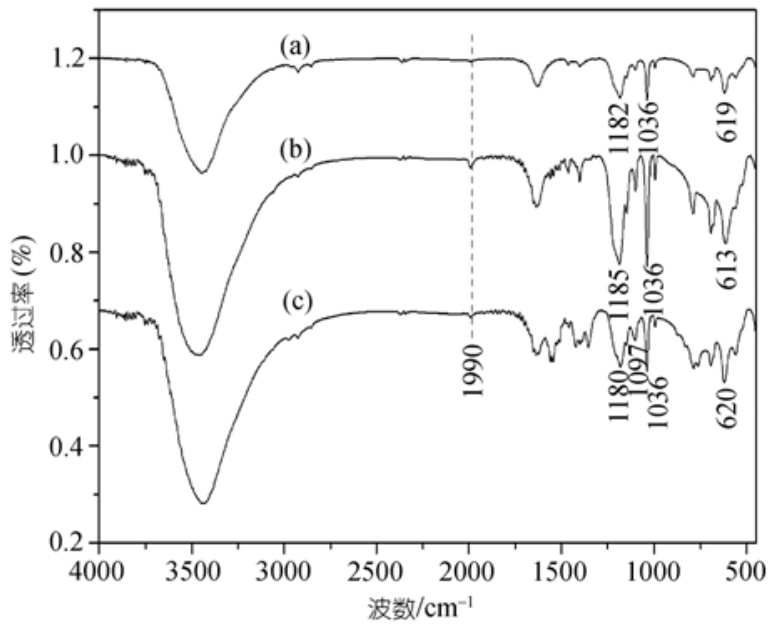

图 5 水溶性铑膦配合物插层 LDHs 样品的红外光谱 (a) $\mathrm{RhP}_{2} / \mathrm{P} / \mathrm{NO}_{3}-\mathrm{Zn}_{2} \mathrm{Al}-\mathrm{LDHs}$, (b) $\mathrm{RhP}_{2} / \mathrm{P} / \mathrm{Cl}-\mathrm{Zn}_{2} \mathrm{Al}-\mathrm{LDHs}$, (c) $\mathrm{RhP}_{2} / \mathrm{P} / \mathrm{SO}_{4}-\mathrm{Zn}_{2} \mathrm{Al}-\mathrm{LDHs}$
$(\mathrm{CO})(\mathrm{TPPTS})_{2}$ 和 TPPTS(图 2(a)和(b))相比未发生位移. 有所不同的是, 样品 $\mathrm{RhP}_{2} / \mathrm{P} / \mathrm{SO}_{4}-\mathrm{Zn}_{2} \mathrm{Al}-\mathrm{LDHs}$ 在 1097 $\mathrm{cm}^{-1}$ 处的吸收峰稍有宽化, 这是由于层间残余 $\mathrm{SO}_{4}^{2-}$ 的 $v_{3}$ 振动吸收峰的存在引起的.

采用 ICP-AES 测定样品的主要元素含量, 并计 算其摩尔比, 结果列于表 5. 本文中的插层 LDHs 样 品的 $\mathrm{Al} / \mathrm{P}$ 摩尔比是考察 $\mathrm{LDHs}$ 有机阴离子插层量的 一个重要数值. 分别以硝酸根 LDHs 和氯离子 LDHs 为离子交换前体的催化剂样品 $\mathrm{RhP}_{2} / \mathrm{P} / \mathrm{NO}_{3}-\mathrm{Zn}_{2} \mathrm{Al}-$ $\mathrm{LDHs}$ 及 $\mathrm{RhP}_{2} / \mathrm{P} / \mathrm{Cl}-\mathrm{Zn}_{2} \mathrm{Al}-\mathrm{LDHs}$ ，与以硫酸根 LDHs 为 离子交换前体的催化剂样品 $\mathrm{RhP}_{2} / \mathrm{P} / \mathrm{SO}_{4}-\mathrm{Zn}_{2} \mathrm{Al}-\mathrm{LDHs}$ 的有较大差别, 后者的 $\mathrm{Al} / \mathrm{P}$ 摩尔比远远大于前两者. 根据 LDHs 主客体层板电荷平衡的原则结合 FT-IR 结 果, 说明样品 $\mathrm{RhP}_{2} / \mathrm{P} / \mathrm{SO}_{4}-\mathrm{Zn}_{2} \mathrm{Al}-\mathrm{LDHs}$ 层间仍然存在 较多硫酸根离子参与平衡层板正电荷. 这可能是由 于 $\mathrm{SO}_{4}^{2-}$ 与 $\mathrm{LDHs}$ 主体层板的静电作用力较强, 导致了 离子交换过程进行不彻底所造成的. 结合 3 个样品的 XRD, FT-IR 及 ICP-AES 结果可以得出, $\mathrm{RhCl}(\mathrm{CO})$ $(\mathrm{TPPTS})_{2}$ 与 TPPTS 已共插层进入锌铝 LDHs 层间，成 功制备了插层 LDHs 催化剂样品.

在相同的烯烃氢甲酰化反应条件下，考察催化剂 样品中客体无机阴离子种类 $\left(\mathrm{RhP}_{2} / \mathrm{P} / \mathrm{NO}_{3}-\mathrm{Zn}_{2} \mathrm{Al}-\mathrm{LDHs}\right.$ 中的 $\mathrm{NO}_{3}^{-}, \mathrm{RhP}_{2} / \mathrm{P} / \mathrm{Cl}-\mathrm{Zn}_{2} \mathrm{Al}-\mathrm{LDHs}$ 中的 $\mathrm{Cl}^{-}, \mathrm{RhP}_{2} / \mathrm{P} /$ $\mathrm{SO}_{4}-\mathrm{Zn}_{2} \mathrm{Al}-\mathrm{LDHs}$ 中的 $\mathrm{SO}_{4}^{2-}$ )对其催化性能的影响, 结 果列于表 6 .

在催化活性方面, $\mathrm{Cl}^{-}$与 $\mathrm{NO}_{3}^{-}$相比, 前者在层间的 共存使得催化活性下降。这是由于 $\mathrm{Cl}^{-}$的存在使得 $\mathrm{RhCl}(\mathrm{CO})(\mathrm{TPPTS})_{2}$ 不利于生成催化活性物种 HRh$(\mathrm{CO})(\mathrm{TPPTS})_{2}$ (图 3 物质 1 ), 因而造成了样品 $\mathrm{RhP}_{2} / \mathrm{P} /$ $\mathrm{Cl}-\mathrm{Zn}_{2} \mathrm{Al}-\mathrm{LDHs}$ 的催化活性较低. 同时样品 $\mathrm{RhP}_{2} /$

表 5 水溶性铑膦配合物插层 LDHs 样品中主要元素的含量及摩尔比

\begin{tabular}{cccc}
\hline 样品 & $\mathrm{RhP}_{2} / \mathrm{P} / \mathrm{NO}_{3}-\mathrm{Zn}_{2} \mathrm{Al}-\mathrm{LDHs}$ & $\mathrm{RhP}_{2} / \mathrm{P} / \mathrm{Cl}-\mathrm{Zn}_{2} \mathrm{Al}-\mathrm{LDHs}$ & $\mathrm{RhP}_{2} / \mathrm{P} / \mathrm{SO}_{4}-\mathrm{Zn}_{2} \mathrm{Al}-\mathrm{LDHs}$ \\
\hline 摩尔比 $\mathrm{Zn} / \mathrm{Al}$ & 2.0 & 2.1 & 2.1 \\
摩尔比 $\mathrm{P} / \mathrm{Rh}$ & 12.7 & 10.0 & 11.8 \\
摩尔比 $\mathrm{Al} / \mathrm{P}$ & 3.3 & 3.3 & 6.3 \\
$\mathrm{Rh}(w / w)(\%)$ & 0.45 & 0.37 & 0.27 \\
\hline
\end{tabular}


表 6 LDHs 层间无机阴离子种类对催化剂样品在 1-己烯氢甲酰化反应中催化性能的影响 a)

\begin{tabular}{cccc}
\hline 样品 & 转化率 $(\%)$ & $\mathrm{TOF} \mathrm{mol}($ 醛 $) \cdot \mathrm{mol}^{-1}(\mathrm{Rh}) \cdot \mathrm{h}^{-1}$ & $n / i$ \\
\hline $\mathrm{RhP}_{2} / \mathrm{P} / \mathrm{NO}_{3}-\mathrm{Zn}_{2} \mathrm{Al}-\mathrm{LDHs}$ & 85.88 & 292.44 & 2.29 \\
$\mathrm{RhP}_{2} / \mathrm{P} / \mathrm{Cl}-\mathrm{Zn}_{2} \mathrm{Al}-\mathrm{LDHs}$ & 76.21 & 171.49 & 2.45 \\
$\mathrm{RhP}_{2} / \mathrm{P} / \mathrm{SO}_{4}-\mathrm{Zn}_{2} \mathrm{Al}-\mathrm{LDHs}$ & 41.08 & 133.46 & 3.18 \\
\hline
\end{tabular}

a) $\mathrm{Rh} / 1$-hexene $=4.0 \times 10^{-4}$, 反应条件: $1 \mathrm{~mL}$ 1-hexene, $1.6 \mathrm{MPa}, 100^{\circ} \mathrm{C}, n\left(\mathrm{H}_{2}\right) / n(\mathrm{CO})=1,6 \mathrm{~h}$ 反应时间

$\mathrm{P} / \mathrm{Cl}-\mathrm{Zn}_{2} \mathrm{Al}-\mathrm{LDHs}$ 中的 $\mathrm{Rh} \%$ 比样品 $\mathrm{RhP}_{2} / \mathrm{P} / \mathrm{NO}_{3}-\mathrm{Zn}_{2} \mathrm{Al}-$ LDHs 稍低(表 5), 这也是导致两者催化活性存在差异 的因素之一. 由表 5 可知, 由于离子交换前体中 $\mathrm{SO}_{4}^{2-}$ 的存在使得 $\mathrm{RhP}_{2} / \mathrm{P} / \mathrm{SO}_{4}-\mathrm{Zn}_{2} \mathrm{Al}-\mathrm{LDHs}$ 样品中催化物质 的负载量(即金属铑含量)最低，因此导致了其催化活 性在 3 个催化剂样品中最低.

就正构醛的催化选择性而言, 样品 $\mathrm{RhP}_{2} / \mathrm{P} / \mathrm{Cl}-$ $\mathrm{Zn}_{2} \mathrm{Al}-\mathrm{LDHs}$ 和 $\mathrm{RhP}_{2} / \mathrm{P} / \mathrm{NO}_{3}-\mathrm{Zn}_{2} \mathrm{Al}-\mathrm{LDHs}$ 的选择性相 近, 而 $\mathrm{RhP}_{2} / \mathrm{P} / \mathrm{SO}_{4}-\mathrm{Zn}_{2} \mathrm{Al}-\mathrm{LDHs}$ 比前两者高. 这是由 于 $\mathrm{RhP}_{2} / \mathrm{P} / \mathrm{SO}_{4}-\mathrm{Zn}_{2} \mathrm{Al}-\mathrm{LDHs}$ 样品的 $\mathrm{Rh} \%$ 最低, 造成层 间的 $\mathrm{RhCl}(\mathrm{CO})(\mathrm{TPPTS})_{2}$ 排布密度最低, 更有利于体 现 LDHs 层板结构的空间选择性. 即空间因素的限制 使得在催化过程中更趋向于生成正构醛的中间体, 从而造成该体系中正构醛的选择性最高.

由表 6 可以得出, 随着催化剂样品活性物质负载 量( $\mathrm{Rh} \%)$ 的上升, 产物醛 $\mathrm{TOF}$ 值也随之上升, $\mathrm{RhP}_{2} / \mathrm{P} /$ $\mathrm{NO}_{3}-\mathrm{Zn}_{2} \mathrm{Al}-\mathrm{LDHs}$ 催化体系出现最高的 TOF 值 $(292$ $\mathrm{h}^{-1}$ ). 除了催化物质负载量的影响因素之外, 这可能
还与层间无机阴离子助催化作用有关, 其原因尚在 进一步研究中.

\section{3 结论}

本文以 TPPTS 插层 LDHs 为前体, 通过原位络合 法制备了 $\mathrm{RhCl}(\mathrm{CO})(\mathrm{TPPTS})_{2}$ 与 TPPTS 共插层 LDHs, 并研究了催化剂样品主、客体组成的改变对其在烯烃 氢甲酰化反应中催化性能的影响. 结果表明, LDHs 主体层板 $\mathrm{M}^{2+}$ 元素电负性的增长导致 LDHs 层板碱性 的降低以及主-客体作用力的增强, 前者使得催化剂 样品的催化活性下降, 后者造成其选择性下降. 层间 共存客体无机阴离子种类 $\left(\mathrm{SO}_{4}^{2-}, \mathrm{Cl}^{-}, \mathrm{NO}_{3}^{-}\right)$对催化剂 样品的催化活性影响较大. 由于 $\mathrm{SO}_{4}^{2-}$ 的共存造成催 化物种负载量降低, 导致催化活性最低(41\%); 由于 $\mathrm{Cl}^{-}$抑制了催化活性物种的生成, 同时该样品中催化 物种的负载量稍低, 使得催化剂样品的催化活性较 低 $(76 \%)$; 而 $\mathrm{NO}_{3}^{-}$在层间的共存未造成以上影响, 其 催化活性最高 $(86 \%)$.

\section{参考文献}

A-Chem, 2004, 218(2): 211-216 $\underline{\text { [doi] }}$

3 Hung K G W, Papadias D, Björnbom P, et al. Reverse-flow operation for application of imperfectly immobilized catalysts. AIChE J, 2003, 49(1): 151-167 [doi]

4 Lenarda M, Storaro L, Ganzerla R. Hydroformylation of simple olefins catalyzed by metals and clusters supported on unfunctionalized inorganic carriers. J Mol Catal A, 1996, 111(3): 203-237[ [doi]

5 Huang L, Kawi S. An active and stable Wilkinson's complex-derived $\mathrm{SiO}_{2}$-tethered catalyst via an amine ligand for cyclohexene hydroformylation. Catal Lett, 2004, 92(1-2): 57-62 [doi]

6 Ichikawa M. Catalytic hydroformylation of olefins over the rhodium, bimetallic Rh-Co, and cobalt carbonyl clusters supported with some metal oxides. J Catal, 1979, 59(1): 67-78[ [doi]

7 Zhu H J, Ding Y J, Yin H M, et al. Supported rhodium and supported aqueous-phase catalyst, and supported rhodium catalyst modified with water-soluble TPPTS ligands. Appl Catal A- General, 2003, 245(1): 111-117 [doi]

8 Cavani F, Trifirò F, Vaccari A. Hydrotalcite-type anionic clays: Preparation, properties and application. Catal Today, 1991, 11(2): $173-301 \underline{\text { [doi] }}$

9 Khan A I, O'Hare D. Intercalation chemistry of layered double hydroxides: Recent developments and applications. J Mater Chem, 2002, 12(11): 3191-3198 [doi]

10 Meyn M, Beneke K, Lagaly G. Anion-exchange reactions of layered double hydroxides. Inorg Chem, 1990, 29(26): 5201—5207 [doi] 
11 Jakupca M, Dutta P K. Thermal and spectroscopic analysis of a fatty acid-layered double-metal hydroxide and its application as a chromatographic stationary phase. Chem Mater, 1995, 7(5): 989-994[doi]

12 Wei M, Yuan Q, Evans D G, et al. Layered solids as a "molecular container" for pharmaceuticalagents: L-tyrosine-intercalated layered double hydroxiedes. J Mater Chem, 2005, 15(11): 1197-1203 [1doi]

13 Khan A I, Lei L, Norquist A J, et al. Intercalation and controlled release of pharmaceutically active compounds from a layered double hydroxide. Chem Commun, 2001, 1(22): 2342-2343[doi]

Tyner K M, Schiffman S R, Giannelis E P. Nanobiohybrids as delivery vehicles for camptothecin. J Control Release, 2004, 95(3): $501-514$ [doi]

Choudary B M, Chowdari N S, Jyothi K, et al. Catalytic asymmetric dihydroxylation of olefins with reusable OsO $\mathrm{O}_{4}^{2-}$ on ion-exchangers: The scope and reactivity using various cooxidants. J Am Chem Soc, 2002, 124(19): 5341 - 5349 [doi]

Bhattacharjee S, Dines T J, Anderson J A. Synthesis and application of layered double hydroxide-hosted catalysts for stereoselective epoxidation using molecular oxygen or air. J Catal, 2004, 225(2): 398 - 407 [doi]

Sels B, Vos D D, Buntinx M, et al. Layered double hydroxides exchanged with tungstate as biomimetic catalysts for mild oxidative bromination. Nature, 1999, 400(6747): 855-857[doi]

谢鲜梅. 锌铝层状复合氢氧化物的合成研究. 无机材料学报, 1999, 14(2): 245-250

谢鲜梅. 层状化合物镍铝水滑石的制备和表征. 无机化学学报, 2000, 16(1): 43-46

Pagano M A, Forano C, Besse J P. Delamination of layered double hydroxides by use of surfactants. Chem Comm, 2000, (1): 91—92

ide-dioctyl sulfosuccinate nanocomposite. J Nanoparticle Res, 2000, 2(3): 293-298[doi]

Clay Miner, 1993, 41(5): 551-557[doi]

Vaysse C, Guerlou-Demourgues L, Delmas C. Thermal evolution of carbonate pillared layered hydroxides with $(\mathrm{Ni}, \mathrm{L})(\mathrm{L}=\mathrm{Fe}, \mathrm{Co})$ based slabs: Grafting or nongrafting of carbonate anions. Inorg Chem, 2002, 41(25): 6905-6913 [doi]

Prevot V, Forano C, Besse J P, et al. Syntheses and thermal and chemical behavior of tartrate and succinate intercalated $\mathrm{Zn}_{3} \mathrm{Al}$ and $\mathrm{Zn}_{2} \mathrm{Cr}$ layered double hydroxides. Inorg Chem, 1998, 37(17): 4293-4301 [doi]

Herrmann W A, Kellner J, Riepl H. Wasserlösliche metallkomplexe und katalysatoren III. Neue wasserlösliche metallkomplexe des sulfonierten triphenylphosphans (TPPTS): Mn, Fe, Ru, Co, Rh, Ir, Ni, Pd, Pt, Ag, Au. J Organomet Chem, 1990, 389(1): 103$128 \underline{\text { [doi] }}$

Kanezaki E. Intercalation of naphthalene-2,6-disulfonate between layers of $\mathrm{Mg}$ and $\mathrm{Al}$ double hydroxide: Preparation, powder X-Ray diffraction, fourier transform infrared spectra and X-Ray photoelectron spectra. Mater Res Bull, 1999, 34(9): 1435-1440[doi]

Kanezaki E. Unexchangeable interlayer anions; Synthesis and characterization of Zn/Al- and Mg/Al-layered double hydroxides with interlayer alizarin red S. J Incl Phenom Macrocyclic Chem, 2003, 46(1-2): 89-95[ [doi]

He Q, Yin S, Sato T. Synthesis and photochemical properties of zinc-aluminum layered double hydroxide/organic UV ray absorbing molecule/silica nanocomposites. J Phys Chem Solids, 2004, 65(2-3): 395-402[doi]

Choy J H, Jung J S, Oh J M, et al. Layered double hydroxide as an efficient drug reservoir for folate derivatives. Biomaterials, 2004, 25(15): 3059-3064[doi]

Dean J A. 兰氏化学手册. 第 2 版. 北京: 科学出版社, 2003

王征，袁刚，周启昭．水溶性铑膦络合催化体系 1-已烯氢甲酰化反应的研究. 石油化工, 1987, 16(10): 691-698

吴已丑，袁刚，周启昭. 用水溶性铑膦络合催化剂制备高碳醛. 石油化工, 1991, 20(2): 79-89

Park M, Lee C, Lee E J, et al. Layered double hydroxides as potential solid base for beneficial remediation of endosulfan-contaminated soils. J Phys Chem Solids, 2004, 65(2-3): 513-516 [doi]

张慧, 齐荣, 刘丽娜, 等. 镁铁双差基复合金属氧化物的可控合成及晶面生长特征研究. 化学物理学报, 2003, 16(1): 45一 50

Choudary B M, Chowdari N S, Kantam M L, et al. Catalytic asymmetric dihydroxylation of olefins with new catalysts: The first example of heterogenization of $\mathrm{OsO}_{4}^{2-}$ by ion-exchange technique. J Am Chem Soc, 2001, 123(37): 9220-9221 [doi]

229(1): 329-333[doi] 